\section{Assessment of HEMS Teams Performance in Out-of- Hospital Cardiac Arrest (OHCA)}

Dr. Przemyslaw Gula, Patryk Rzonca, Robert Galazkowski, Marcin Podgórski

Polish Air Rescue Service, Krakow, Poland

Introduction: The Out of Hospital Cardiac Arrest (OHCA) procedures constitute one of the most quantifiable indicators of the quality of Emergency Medical Services (EMS). In Poland, HEMS teams perform such procedures both during primary missions and when they support EMS teams.

Aim: To carry out a retrospective analysis of OHCA related calls received from January 1, 2011, to December 31, 2016.

Methods: During the relevant period there were 2,447 OHCA related calls. Of those, 308 cases were excluded from the analysis because no cardiac arrest was confirmed or the patients showed signs of death that prevented any emergency procedures.

Results: The Return of Spontaneous Circulation (ROSC) was achieved in 1,119 cases. Resuscitation was clearly much more effective if CPR procedures were commenced prior to the arrival of the HEMS team. The groups in which higher survival rates were obtained included women, patients younger than 40 and patients who had signs of shockable rhythms. The use of HEMS team allowed for faster transport of patients to relevant specialist institutions, specifically if an invasive cardiological intervention was needed.

Discussion: The use of HEMS teams in OHCA related calls indicates that such actions are highly effective both in primary missions and when HEMS teams support other EMS terms. An additional advantage is the possibility of quick transportation to a relevant specialist medical center.

Prehosp Disaster Med 2019;34(Suppl. 1):s104

doi:10.1017/S1049023X19002152

\section{Availability of Essential Medical Equipment for Prehospital Trauma Care on Public Ambulances in Ukraine \\ Mr. Stanislav Gaiervskyi, Dr. Colin Meghoo \\ Patriot Defence, Kyiv, Ukraine}

Introduction: The public ambulance system in Ukraine is the primary deliverer of prehospital care for trauma patients in this Eastern European country, but no national assessment has previously been made to ensure the presence of essential medical equipment on these ambulances.

Aim: Working with the Ukraine Ministry of Health, our aim was to assess the availability of public ambulances of medical equipment essential for managing traumatic injury using an internationally recognized standard for prehospital care.

Methods: We identified 53 Advanced Life Support (ALS) ambulances from randomly selected cities for evaluation. We performed an inventory of available medical equipment and supplies on these ambulances against a matrix of essential equipment for prehospital providers developed by the World Health Organization (WHO).

Results: Essential medical equipment in the categories of personal protection, patient monitoring, hemorrhage control, and immobilization were generally available in the ALS public ambulances surveyed. Deficiencies were noted in equipment and supplies for basic and advanced airway monitoring and management.

Discussion: Public ALS ambulances across Ukraine are adequately equipped with many essential medical supplies to manage traumatic injury, but have deficiencies in both basic and advanced airway management. Correcting these deficiencies may improve prehospital survival of the traumatically injured patient. The results of this study will enable the Ukraine Ministry of Health to develop requirements of essential medical equipment for all public ALS ambulances in the country, to inform resource allocation decisions, and to guide public health policy regarding prehospital trauma care.

Prehosp Disaster Med 2019;34(Suppl. 1):s104

doi:10.1017/S1049023X19002164

\section{Awareness, Perspective, and Reasons Behind Patients' Rejection of Emergency Medical Services in Thailand Mr. Teera Sirisamutr, Mrs. Porntip Wachiradilok}

National Institute for Emergency Medicine, Nonthaburi, Thailand

Introduction: Emergency Medical Service (EMS) increases survival rates and reduces possible disability among emergency patients. However, the number of requests is relatively low in Thailand.

Aim: To inspect the awareness, perspective, and reasons behind the rejection of EMS by patients or their relatives who visit the emergency room.

Methods: Responses were analyzed in 45 government, university, and private hospitals from December 2015 to February 2016. The hospitals were scattered in 7 provinces with the sample group including 2,028 patients, whereby 646 patients visited using EMS and 1,368 did not. The key reasons for self-visit or other means are the convenience of personal transportation (76.0\%), not wanting to wait for an ambulance (31.0\%), and anxiety caused by the emergency situation (28.9\%). Most misconceptions about the service include; 1) Ambulances are used only for casualties from accidents and 2) Ambulance service are not free. In terms of perspective, most patients or relatives hold a negative view towards the emergency medical service, especially the idea that they can help themselves when the condition is not severe or if there are medications or relief devices available. Another view is that the service will delay them from getting to the hospital. These perspectives are from non-users.

Discussion: The study indicated that the cause of non-user involved misunderstandings, poor perspectives, lack of awareness, and the ignorance of the threat of the particular emergency condition. Thus, they do not realize the benefit of using EMS. As a result, regional agencies, the National Institute of Emergency Medicine, and the Ministry of Public Health should discuss the solutions to raise public awareness and improve the perspective towards emergency medical services to promote more usage.

Prehosp Disaster Med 2019;34(Suppl. 1):s104

doi:10.1017/S1049023X19002176

\author{
Back to the Future: Portable Word Processors and the \\ Electronic Health Record \\ Dr. Alfredo Mori \\ St John Of God Health Care, Geelong, Australia
}


Introduction: The Electronic Health Record (EHR) is now the standard means for recording and maintaining medical notes in most emergency departments. The EHR is an independent cause of physician burnout, and maintenance of the EHR may occupy 30 to $50 \%$ of clinical time. There are software solutions available, but they are connected to fixed, expensive, distracting, and bright electronically powered computers. Scribes have been successfully trialed, but are also expensive and attached to computers on wheels. Portable digital word processors in the form of the AlphaSmart Neo is a redundant technology designed primarily for children with typing difficulties. It has recently enjoyed a resurgence in popularity among professional writers, journalists, and field researchers for the ultimate distraction-free writing experience. The Alphasmart Neo is cheap, nearly indestructible, intuitive, and requires almost no recharging. It is compatible with all software across Mac OS, Windows, and Linux. Notes are entered by the clinician or scribe, independently of computers, at the bedside, and uploaded to any software via USB cable.

Aim: To describe the introduction and impact of the AlphaSmart Neo on the EHR in emergency departments across Australia.

Methods: We will examine the role of the Alphasmart Neo in austere, low power, extreme environments with a demonstration on how to enter, maintain, and transfer an electronic health record independent of any computer or power source.

Discussion: We believe the AlphaSmart Neo is an ideal, personalized, cheap, effective, and efficient hardware solution to entering notes independent of other software and hardware. It is distraction free at the patient's bedside, resulting in better notes that the clinician enjoys writing.

Prehosp Disaster Med 2019;34(Suppl. 1):s104-s105

doi:10.1017/S1049023X19002188

\section{Basic Principles in Complex Humanitarian Emergency: A} Pilot Course

Dr. Nidaa Bajow ${ }^{1}$, Dr. yousef Alawad ${ }^{2}$, Dr. Samer Aloraifi ${ }^{1}$

1. Mohammad Bin Naif Medical Center - king Fahad Security Collage, RIYADH, Saudi Arabia

2. king Fahad Medical city - Minister Of Health, RIYADH, Saudi Arabia

Introduction: The international and national response team faces many challenges during a complex humanitarian emergency. These include difficult organization, an unprepared national disaster plan, and a disrupted political system. Previous studies showed a reactive approach in earlier disasters in Saudi Arabia and the need for greater involvement of health professionals in disaster management. As a result, several medical education and training institutes began to introduce courses which were mainly about Major Incidents Response, but with less attention to Humanitarian Assistance and Disaster Relief. Aim: The course provides Basic Principles in Complex Humanitarian Emergency for healthcare providers in the kingdom and is focused on the aspect of community awareness for disaster and humanitarian relief.

Methods: The interactive competencies-based course in Basic Principles of Complex Humanitarian Emergency was implemented. The course was designed by 5 experts in disaster medicine and humanitarian relief and was piloted over five days at officers club of Minister of Interior in Riyadh, sponsored by King Fahd Security College. The participants $(n=30)$ were from different health disciplines. They completed the pre- and posttests and presented three pilot workshops for disaster community awareness.

Results: The overall scores were $44.19 \%$ for the pre-test and $62.85 \%$ for the post-test (Wilcoxon test for paired sample: $\mathrm{z}=3.729, \mathrm{p}<0.001)$. There were no significant statistical differences among professions of healthcare providers for both pre- and post-tests.

Discussion: Delivering competencies-based course in Basic Principles of Complex Humanitarian Emergency for health care providers can help in the improvement of knowledge and skills for humanitarian assistance and disaster relief in Saudi Arabia, which is important for disaster preparedness augmentation in the kingdom. The next course for the same group may be recommended for achieving the level that will train them to participate in the National Disaster Assistance team.

Prehosp Disaster Med 2019;34(Suppl. 1):s105

doi:10.1017/S1049023X1900219X

\section{Behavioral Health Resource Utilization of Emergency Department Patients Presenting from Mass Gathering Events}

Dr. Charles Hebert, Dr. Gary Peksa, Dr. Joshua DeMott

Rush University Medical Center, Chicago, Ilinois, USA, Chicago, United States

Introduction: Behavioral health needs of attendees at mass gathering events who require emergency department (ED) evaluation are poorly understood. Appropriate resource allocation of mental health staff and other behavioral interventions necessary to support this patient population are also unclear.

Aim: To describe behavioral characteristics and psychiatric resource utilization of patients presenting to a tertiary academic medical center emergency department from mass gathering events.

Methods: Single-center retrospective study evaluating attendees at mass gathering events who presented to a Chicago ED. Electronic medical records for patients presenting between October 13, 2013, and December 31, 2015, were reviewed and descriptive analyses performed.

Results: 209 distinct records were reviewed. Most patients presented from large outdoor concerts $(n=186,89 \%)$. Forty-two (20.1\%) reported a mental health complaint at presentation, including concerns related to pre-existing psychiatric disturbances or onset of new symptoms. Twenty-seven of the total cohort (12.9\%) endorsed a prior psychiatric history. Thirty-five (16.7\%) reported use of prescribed psychotropic medications, including antidepressants, stimulants, mood stabilizers, and others. Diagnostic testing among the total sample included serum ethanol measurement (31.1\%), urinary toxicology (25.4\%), acetaminophen (6.2\%), aspirin (5.3\%), and creatine kinase measurements (11\%). Computed brain tomography was ordered for 20 patients (9.6\%). Twelve patients (5.7\%) 